\title{
RBF-BASED LASER SPECKLE PATTERN DIGITAL IMAGE CORRELATION METHOD FOR SURFACE STRAIN MEASUREMENTS
}

\author{
EDUARDO DIVO ${ }^{1}$, FAISAL MOSLEHY $^{2} \&$ ALAIN KASSAB $^{2}$ \\ ${ }^{1}$ Embry-Riddle Aeronautical University, Mechanical Engineering Department, USA \\ ${ }^{2}$ University of Central Florida, Mechanical and Aerospace Engineering Department, USA
}

\begin{abstract}
This paper introduces an innovative technique that integrates a genetic algorithm based digital image correlation with laser speckle photography (LSP) for the measurement of surface displacements in structures. The images (before and after deformation) are digitized using a digital camera, and the grayscale intensity matrices are read and processed by Matlab image processing toolbox. The two matrices of the images are then inputted into an iterative program based on the genetic algorithm that utilizes an advanced cross correlation technique to determine the surface displacements. Additionally, the strains are computed by radial basis function (RBF) differentiation. The computed displacements are compared with simulated results obtained by finite element analysis. Close agreement between the two results proved the validity of the developed non-contact technique for accurately measuring surface displacements. The experimentally measured displacements can be directly used in an inverse technique to detect and characterize subsurface cavities in structures.
\end{abstract}

Keywords: laser speckle pattern, RBF interpolation, genetic algorithm, surface strain.

\section{INTRODUCTION}

Optical methods such as holographic interferometry, shearography, laser speckle interferometry and Moiré were developed for measuring surface displacement and deformation. Some of these methods have been combined with the latest computer technology and imaging systems and developed into commercial scientific instruments. In practice, however, this equipment is expensive and requires a stable environment as well as laborious data reduction processes. References [1]-[4] are resourceful in literature and description of the laser speckle interferometry and photography techniques. Fraley et al. [5] proposed the use of the two-dimensional correlation method to measure the local displacement with laser speckle patterns. This approach has been applied in several special cases to determine the local displacement components due to uniform translation in pixel resolution.

A more straightforward technique using digital image correlation method was developed in the early 1980s for measuring surface displacements and deformation [6]. The method required a digital imaging system to optically record the images of the surface before and after deformation. The gray level functions of the images are then compared using advanced image correlation and processing algorithms in order to determine the displacement and deformation gradients. Pilch et al. [7] and Chu et al. [8] developed a new optimizing algorithm based on evolutionary techniques for digital image correlation. The optimizing process was tested for a rubber specimen under tensile load using speckle formed by spray painting the specimen surface.

In this paper, we developed an image domain decomposition approach to laser speckle photography (LSP) as applied to the vision-based method of deformation measurements. Here, a genetic algorithm is utilized to determine the motion of the center of pixel groupings by maximizing a simplified cross correlation expression. The whole specimen 
deformation is subsequently reconstructed by means of radial basis function interpolation $(\mathrm{RBF})$, and the strains are found by RBF differentiation.

\section{THE DOMAIN DECOMPOSITION LASER SPECKLE TECHNIQUE}

The application of the LSP technique involves the use of speckle pattern formed on the surface of a specimen impinged by a coherent source of light such as a laser beam. The procedure entails the application of a random speckle pattern to a surface of interest, then capturing a digital image of the surface, followed by capturing an image of the specimen after the surface has been displaced or deformed and then comparing the two images to compute the displacement for the surface. A schematic of the experimental setup is shown in Fig. 1.

Suppose a speckle pattern is created on a surface and an image is captured, as depicted in Fig. 2(a). A point of interest in this image undergoes motion in the second image (Fig. 2(b)) depending upon the loading conditions. A region (subset) around the point of interest may be selected. This subset may undergo a displacement and strain such that the new location and shape of the subset is depicted by the parallelogram in Fig. 2(b). The center of the parallelogram is the point of interest.

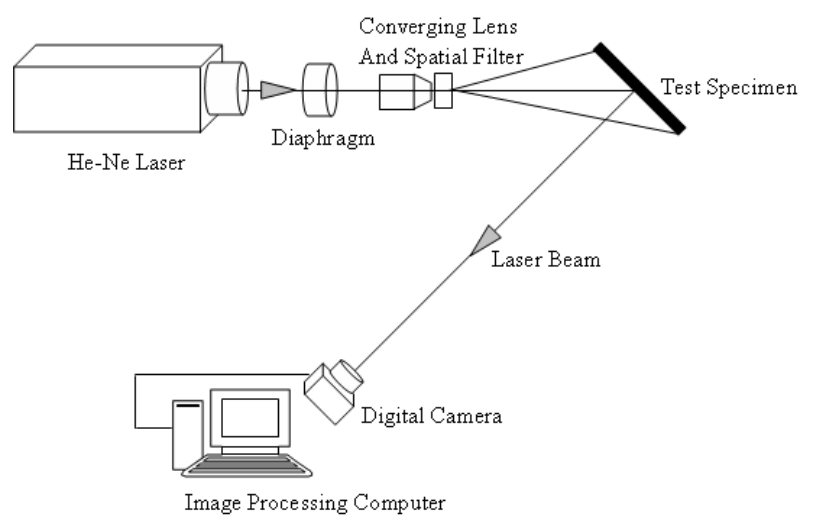

Figure 1: Schematic of the experimental setup.
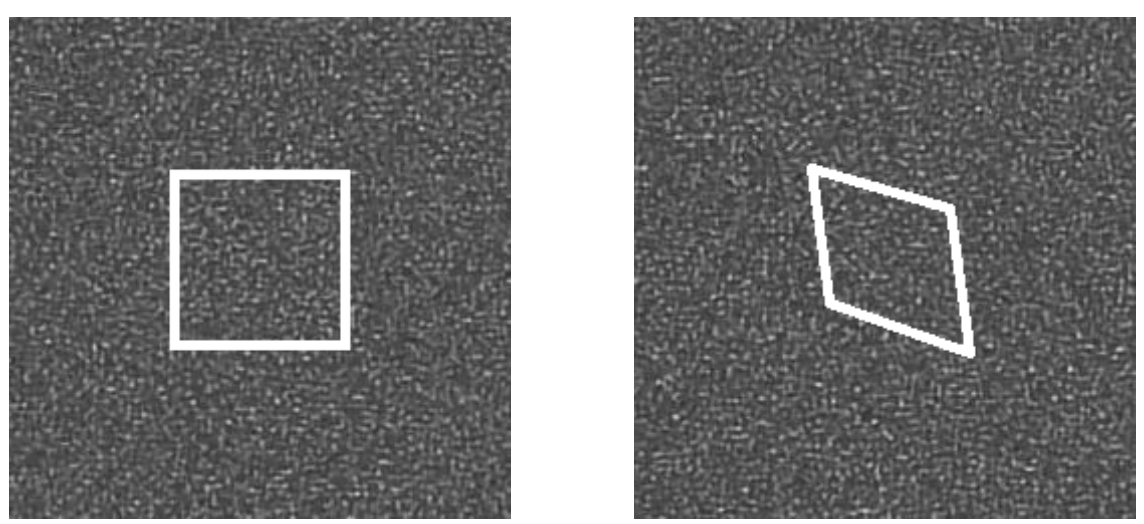

Figure 2: (a) Undeformed image; (b) Actual deformed image. 


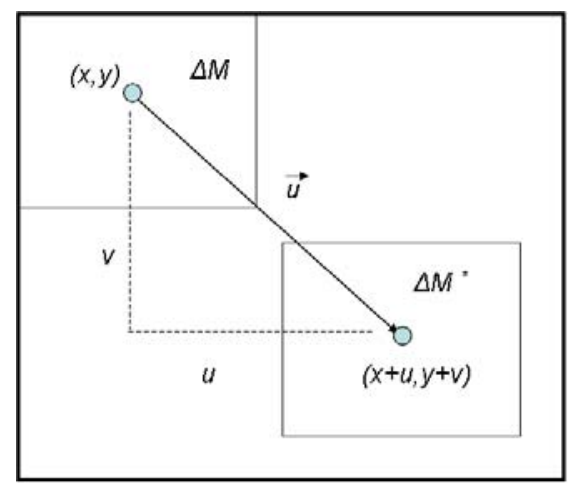

Figure 3: Model utilized in this study: motion of the center of a grouping of pixels.

The method for comparing the two subsets (to determine the displacement vector $\vec{u}$, see Fig. 3) is commonly given by the cross correlation coefficient, C:

$$
C(u, v)=\frac{\int_{\Delta M^{*}} f(x, y) f^{*}(x+u, y+v) d A}{\sqrt{\left(\int_{\Delta M} f^{2}(x, y) d A\right)\left(\int_{\Delta M} f^{* 2}(x+u, y+v) d A\right)}},
$$

where: $\Delta M=$ subset in the undeformed image;

$\Delta M^{*}=$ subset in the deformed image;

$f(x, y)=$ grey level of speckle at a point (pixel).

The values of $u$ and $v$ which maximize $\mathrm{C}$ are the local deformation (displacement components) for the selected subset. The main objective of the image correlation process is to find these values for the subset under investigation, and then repeat it for all subsets in a given region to find the whole field deformation profile. In practice, the above is then implemented as:

$$
C(u, v)=\frac{\left(\sum_{\Delta M}(\text { grey levels })\right)\left(\sum_{\Delta M^{*}}(\text { grey levels })\right)}{\left(\sum_{\Delta M}(\text { grey levels })^{2}\right)\left(\sum_{\Delta M^{*}}(\text { grey levels })^{2}\right)} .
$$

Generally, the above correlation should be at least augmented by first derivatives to account for actual deformations not just translation. However, we since adopt a domain decomposition approach to the problem, the deforming regions that are followed are significantly reduced in size with each subdomain viewed with the full resolution of the camera as illustrated in Fig. 4. For each grouping of pixels, the values of $u$ and $v$ that maximize the correlation in eqn (1) are determined by a genetic algorithm (GA). The image of the whole specimen is then reconstructed via Radial Basis function (RBF) interpolation of the motion of each pixel grouping. 


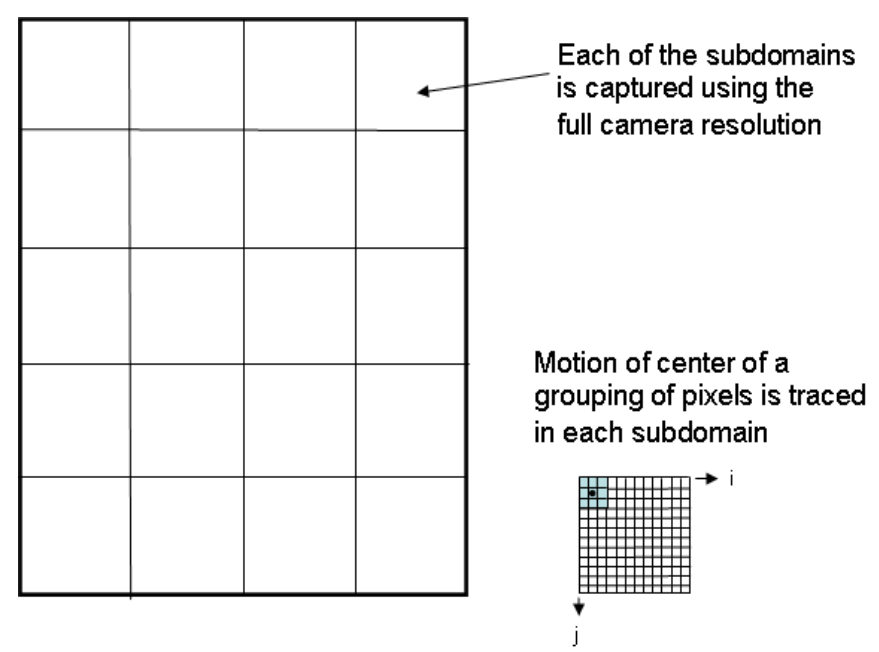

Figure 4: Domain decomposition of the image.

\section{THE GENETIC ALGORITHM}

The genetic algorithm (GA) optimization process begins by setting up a random set of possible solutions, called the population, with a fixed initial size or number of individuals. Each individual is defined by optimization variables and is represented as a bit string or a chromosome. An objective function, in our case $Z(u, v)=1 / C(u, v)$, is evaluated for every individual in the current population defining their fitness or their probability of survival. At each of the iterations of the GA, the processes of selection, cross-over, and mutation operators are used to update the population of designs. A selection operator is first applied to the population in order to determine and select the individuals that are going to pass information in a mating process with the rest of the individuals in the population. This process allows the genetic information contained in the best individuals to be combined to form offspring. Additionally, a mutation operator randomly affects the information obtained by the mating of individuals. This is a crucial step for continuous improvement.

In nature, the properties of an organism are described by a string of genes in the chromosomes. Therefore, if one is trying to simulate nature using computers one must encode the design variable in a convenient way. We adopt a haploid model using a binary vector to model a single chromosome. The length of the vector is dictated by the number of design variables and the required precision of each design variable. Each design variable has to be bounded with a minimum and a maximum value and in the process the precision of the variable is determined. The number of divisions used in the discretization has to be integer power of two. This procedure allows an easy mapping from real numbers to binary strings and vice versa. The haploid GAs place all design variables into one binary string. In turn, each individual is equipped with a given set of design variables to which corresponds a value of the objective function. This value is the measure of "fitness" of the individual design. In GAs, poorly fit designs are not discarded; rather they are kept, as in nature, to provide genetic diversity in the evolution of the population. This genetic diversity is required to provide forward movement of the population during the mating, cross-over, and mutation processes which characterize the GA (see Goldberg [9]). 
After initializing the population with random values the fitness of each individual is calculated by computing the value of the objective function. Then, the probability of being a selected individual for mating is calculated as the ratio between the value of the fitness function of each individual and the sum of all fitness function values. This is given by:

$$
P_{\text {selescted }}=\frac{\text { Fitness }\left(v_{i}\right)}{\sum_{i=1}^{\text {population-size }} \operatorname{Fitness}\left(v_{i}\right)} .
$$

where $v_{i}$ is $i$ th member of the population, and Fitness $\left(v_{i}\right)$ is the measure of the fitness of that member under its currently evolved parameter set configuration. A weighted roulette wheel is generated, where each member of the current population is assigned a portion of the wheel in proportion to its probability of selection. The wheel is spun as many times as there are individuals in the population to select which members mate. Obviously, some individuals could be selected more than once, where the best chromosomes get more copies, the average stay even, and the worst die off. Once selection has been applied, crossover and mutation occur to the resulting offspring. These operations further expand genetic diversity in the population. All other probabilities referred to in the description of the GA adopted in this paper are computed in an analogous fashion as the selection probability.

Following selection, crossover and mutation the new population is ready for its next evolution until the convergence criteria "fitness" is reached. It is the very nature of the binary representation of the design variables of the objective function and the random search process which provide yet another but implicit degree of regularization in this optimization process. The sensitivity of the objective function can be tuned depending on the size of each element of the chromosome. Thus, low bit representation is insensitive to large variations in input (regularized but may lead to poor solution due to low resolution), while high bit representation is sensitive to large variations in input (not regularized and therefore may lead to poor solution as well). There is a range of bit size which produces a regularized and sensitive response leading to stable solutions.

In the GA we used to generate the results presented in this paper, the following parameters are chosen: population size of individuals/generation, with a string of ten bits to define each parameter within each individual, two offspring per mating, a $1 \%$ probability of mutation, and a $70 \%$ probability of crossover. The population is not allowed to grow. This combination of parameters has been proven to yield efficient and accurate optimization results for different studies carried out by the authors [10].

\section{RBF INTERPOLATION AND STRAIN COMPUTATION}

The radial basis function (RBF) interpolation begins by defining a set of data centers, $x_{c}$, comprised of points distributed throughout the domain and the boundary which need not be uniformly distributed. These data centers serve as collocation points for the localized expansion of the variables of interest in the domain and on the boundary.

A localized expansion over a group or topology of influence points, $N F$, around each data center is sought such that:

$$
\phi(x)=\sum_{j=1}^{N F} \alpha_{j} \chi_{j}(x)+\sum_{j=1}^{N P} \alpha_{j+N F} P_{j}(x) .
$$


The variable $\varphi$ will take on the values of $u$ or $v$ depending on the application, $x$ denotes the $x-y$ pair of coordinates of a general point, $\alpha_{j}$ denote the unknown expansion coefficients, $\chi_{j}$ are the RBF expansion functions, $N P$ is the number of additional polynomial functions, $P_{j}(x)$, added to the expansion to guarantee that constant and linear fields can be retrieved exactly. The RBF we utilize belong to the family of Hardy Multiquadrics [11]:

$$
\chi_{j}(x)=\left[r_{j}^{2}(x)+c^{2}\right]^{n-\frac{3}{2}},
$$

where, $n$ is a positive exponent, $c$ is a shape parameter, and $r_{j}(x)$ is the Euclidean distance from $x$ to $x_{i}$. We utilize $\mathrm{n}=1$, a choice that yields the well-established inverse Multiquadrics, whose behavior has been extensively studied in the literature [12], [13]. The shape parameter, $c$, controls the behavior of the interpolation and the accuracy of its derivative. For a specific expansion over a set of data centers, the larger the shape parameter, the smoother is the computed derivative field. However, the magnitude of the shape parameter cannot be increased without bound as the expansion functions become flatter and, hence, the collocation coefficient matrix becomes ill-conditioned. For this reason, a simple optimization search is employed to determine every value for the shape parameter used in every expansion over the different local topologies that cover the entire field. An initial guess for $c$ is based on the ratio of the average distance between data centers in a topology to the number of points in the topology.

The localized expansion approach [14], [15] reduces the computational burden global RBF interpolation methods by expanding the field variable locally around each data center to interpolation the function and to obtain its derivatives. This approach yields the generation of a different but small interpolation matrix for each data center rather than the large and fully-populated global interpolation matrix of the standard global interpolation methods.

The selection of an influence region or localized topology of expansion around each data center is easily accomplished by a circular (spherical in 3D) search around each data center. The search is automated to guarantee that a minimum number of points will be included and additional criteria, such as including all directions around internal data centers, are met. The collocation of the field variable at the points within the localized topology, leads to the following in matrix-vector form:

$$
\{\phi\}=[C]\{\alpha\} \Rightarrow\{\alpha\}=[C]^{-1}\{\phi\},
$$

where the matrix $[\mathrm{C}]$ and the vector $\{\varphi\}$ are composed as:

$$
[C]=\left[\begin{array}{cccccc}
\chi_{1}\left(x_{1}\right) & \ldots & \chi_{N F}\left(x_{1}\right) & P_{1}\left(x_{1}\right) & \ldots & P_{N P}\left(x_{1}\right) \\
\cdot & . & \cdot & \cdot & . & \cdot \\
\chi_{1}\left(x_{N F}\right) & \ldots & \chi_{N F}\left(x_{N F}\right) & P_{1}\left(x_{N F}\right) & \ldots & P_{N P}\left(x_{N F}\right) \\
P_{1}\left(x_{1}\right) & \ldots & P_{1}\left(x_{N F}\right) & 0 & \ldots & 0 \\
\cdot & . & . & . & . & . \\
P_{N P}\left(x_{1}\right) & \ldots & P_{N P}\left(x_{N F}\right) & 0 & \ldots & 0
\end{array}\right] \text { and }\{\varphi\}=\left\{\begin{array}{c}
\varphi\left(x_{1}\right) \\
\cdot \\
\varphi\left(x_{N F}\right) \\
0 \\
. \\
0
\end{array}\right\}
$$

and, evaluating eqn (4) for field variable at the data center $x_{c}$ leads to the matrix form:

$$
\phi\left(x_{c}\right)=\left\{\chi\left(x_{c}\right)\right\}^{T}[C]^{-1}\{\phi\}=\left\{\psi\left(x_{c}\right)\right\}^{T}\{\phi\} .
$$



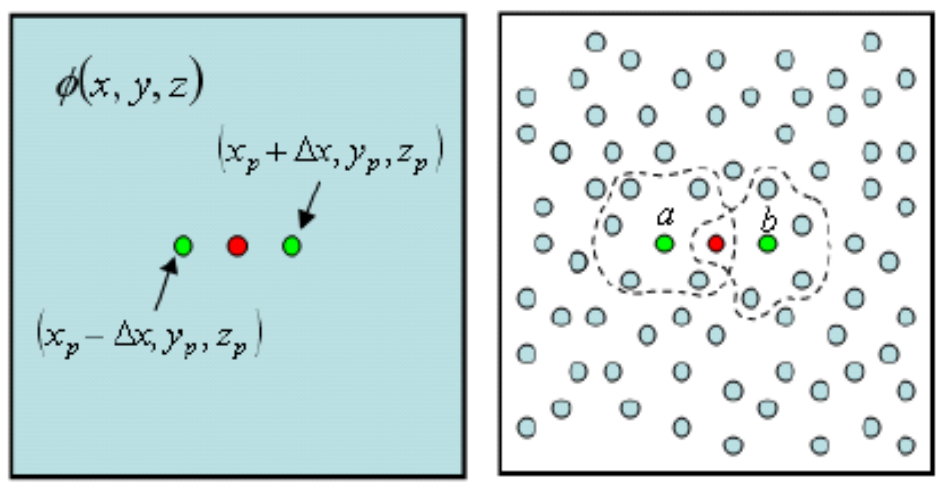

Figure 5: Differentiation stencil and local topology for RBF-FD.

To compute variable derivatives at the data center there are two possibilities: (1) RBF native differentiation (RBF-ND) we apply any linear differential operator, for example, $L_{x}=\partial / \partial x$, over the localized expansion equation, or (2) RBF-enhanced finite difference (RBF-FD) where we utilize the RBF to interpolate the field variable at locations on a finite difference stencil (virtual points) centered at the data center, and subsequently apply a standard finite difference on that stencil. In the first case of RBF-ND, the approach leads to:

$$
L \phi\left(x_{c}\right)=\sum_{j=1}^{N F} \alpha_{j} L \chi_{j}\left(x_{c}\right)+\sum_{j=1}^{N P} \alpha_{j+N F} L P_{j}\left(x_{c}\right),
$$

where $x_{c}$ is the data center of the topology. Thus, in matrix-vector form:

$$
L \phi_{c}=\left\{L_{c}\right\}^{T}\{\alpha\} \Rightarrow L \phi_{c}=\left\{L_{c}\right\}^{T}[C]^{-1}\{\phi\} \Rightarrow L \phi_{c}=\{L\}^{T}\{\phi\},
$$

where the vector $\left\{L_{c}\right\}$ is composed by:

$$
\left\{L_{c}\right\}=\left(\begin{array}{c}
L \chi_{1}\left(x_{c}\right) \\
\vdots \\
L \chi_{N F}\left(x_{c}\right) \\
L P_{1}\left(x_{c}\right) \\
\vdots \\
L P_{N P}\left(x_{c}\right)
\end{array}\right) .
$$

In the second case of RBF-FD, illustrated for the second order central difference evaluation of the first derivative at the data center $x_{p}$, the RBF is utilized to generate the values for the field variable at the virtual finite difference stencil locations $a$ and $b$. Local topologies are constructed for each virtual data center and these do not include the virtual points themselves, see Fig. 5. Eqn (6) is applied to the set of points surrounding the virtual points $a$ and $b$, leading to:

$$
\phi\left(x_{a}\right)=\left\{\psi\left(x_{a}\right)\right\}^{T}\{\phi\}_{a},
$$




$$
\phi\left(x_{b}\right)=\left\{\psi\left(x_{b}\right)\right\}^{T}\{\phi\}_{b},
$$

where $\left\{\psi\left(x_{a}\right)\right\}$ is the interpolating weight vector and $\{\phi\}_{a}$ is the nodal value vector associated with virtual point $a$, and $\left\{\psi\left(x_{b}\right)\right\}$ is the interpolating weight vector and $\{\phi\}_{b}$ is the nodal value vector associated with virtual point $b$.

Therefore, substituting these virtual point expressions into a second order accurate central difference of the first $\mathrm{x}$-derivative at the data center $x_{c}$ leads to:

$$
\frac{\partial \phi}{\partial x}=\left\{\frac{1}{2 \Delta x}\left\{\psi\left(x_{a}\right)\right\}^{T} \frac{-1}{2 \Delta x}\left\{\psi\left(x_{b}\right)\right\}^{T}\right\}^{T}\left\{\begin{array}{l}
\left\{\phi_{a}\right\} \\
\left\{\phi_{b}\right\}
\end{array}\right\}=\left\{L_{c, x}\right\}^{T}\{\phi\} .
$$

This is readily extended for any other derivative of interest. Therefore, using either method, RBF-ND or RBF-FD, the evaluation of the field variable derivatives at every one of the data centers $x_{c}$ is provided by a simple inner product of two small vectors: $\left\{L_{c}\right\}$ which can be pre-built and stored and $\{\phi\}$ which is the field variable values at the surrounding RBF points within the topology of the data center $x_{c}$. In this paper, we compute all derivatives utilizing the RBF-FD. With the derivatives of the displacements now readily available, the strains can be computed as:

$$
\varepsilon_{x}=\frac{\partial u}{\partial x}, \varepsilon_{y}=\frac{\partial v}{\partial y}, \gamma_{x y}=\frac{\partial u}{\partial y}+\frac{\partial v}{\partial x}
$$

\section{EXPERIMENTAL PROCEDURE AND DATA COLLECTION}

A Plexiglas test specimen of length $=250 \mathrm{~mm}$, width $=168 \mathrm{~mm}$, and thickness $=4.8 \mathrm{~mm}$, with two circular holes was fabricated and all the optical instruments were clamped firmly onto an optical table (see Fig. 6). The translation stage holding the spatial filter was adjusted till a clean divergent laser beam is obtained to illuminate the test specimen. The test specimen was then clamped onto the loading frame and a tensile load of $500 \mathrm{lb}$ was applied. An image of the specimen (covered by laser speckles) in the deformed state was captured by a digital camera. Subsequently the specimen was unloaded to $100 \mathrm{lb}$ and another digital image was captured.
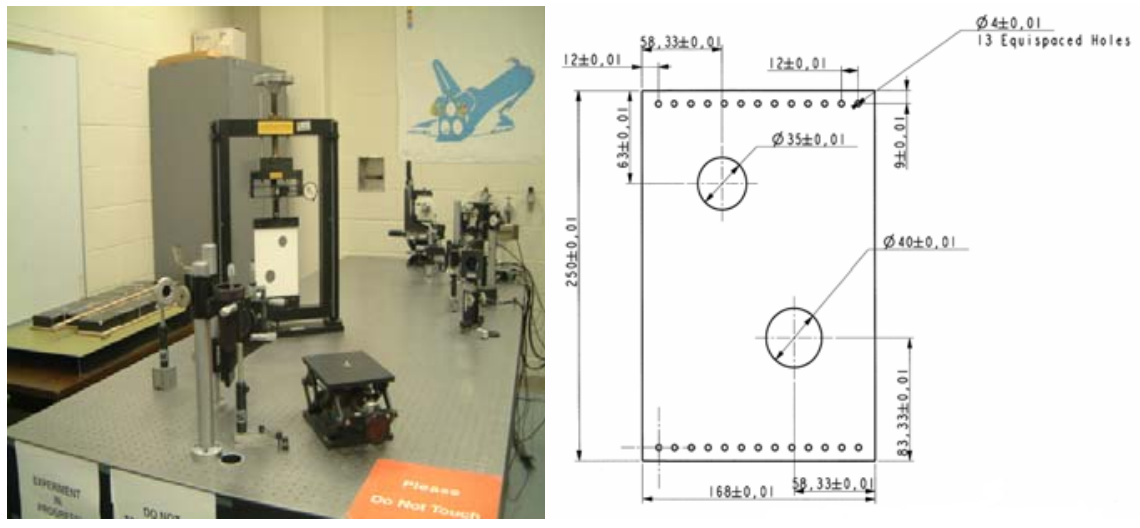

Figure 6: Photograph of the experimental setup and details of the test specimen. 
The digital camera at highest resolution and full zoom can give resolution of $2832 \mathrm{x}$ 2128 pixels. Once the digital images are cropped to size, the resolution for the images was calculated to be $0.125 \mathrm{~mm} /$ pixel. Fig. 7 depicts the gray level distribution. Preliminary finite element analysis indicated that the maximum deformation would be in the order of $0.3 \mathrm{~mm}$, or in terms of pixels the maximum expected deformation would be less than 3 pixels. Preliminary runs of the image correlation using the genetic algorithm proved that such low-resolution images gave poor output for surface displacements.

To overcome the low-resolution problem it was then decided to subdivide the entire test specimen into blocks and capture the image of each block separately at highest resolution and full zoom. As shown in Fig. 8(a) and (b) the specimen surface was divided into an array of 20 blocks using a soft-tipped pen and each block was identified by its own numbering scheme.

The camera was mounted on a translation stage firmly clamped onto the isolation mount and placed $250 \mathrm{~mm}$ away from the test specimen. The camera was set at the highest resolution and zoomed to focus on the first block of the specimen. The specimen was

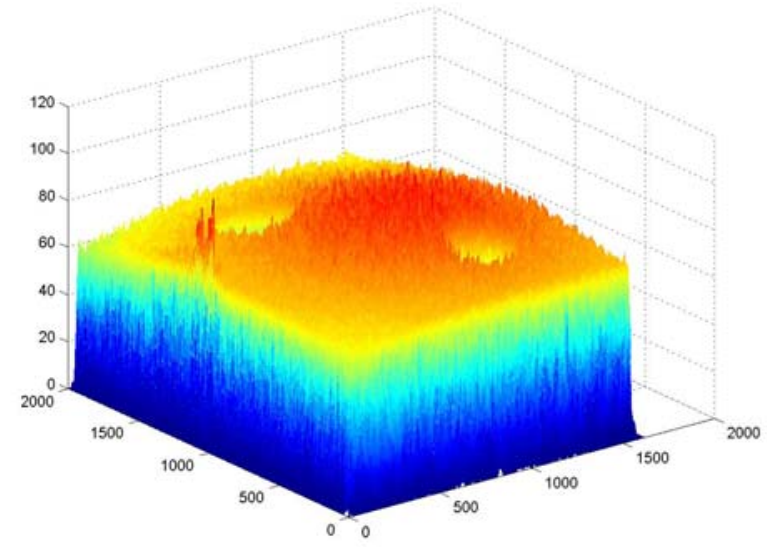

Figure 7: Grey level distribution of specimen covered by laser speckles.

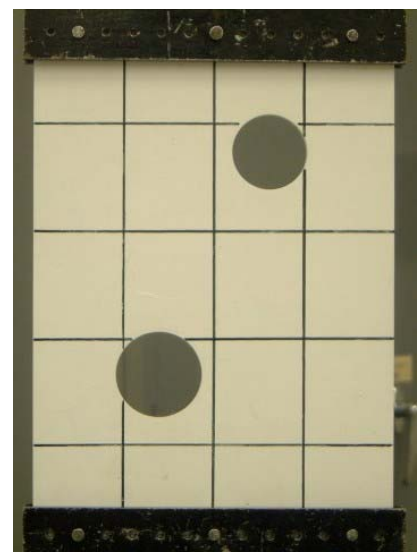

\begin{tabular}{|c|c|c|c|}
\hline 11 & 12 & 13 & 14 \\
\hline 21 & 22 & 23 & 24 \\
\hline 31 & 32 & 33 & 34 \\
\hline 41 & 42 & 43 & 44 \\
\hline 51 & 52 & 53 & 54 \\
\hline
\end{tabular}

Figure 8: (a) Subdivided specimen; and (b) Numbering scheme. 
loaded to $500 \mathrm{lb}$; an image captured and subsequently the specimen was unloaded to $100 \mathrm{lb}$ and another image captured. The resolution obtained by this technique was found to be $0.027 \mathrm{~mm} /$ pixel. This procedure was repeated for the twenty blocks of the specimen. The image intensity matrix for each set was correlated using the genetic algorithm-based correlation technique. The input parameters of the algorithm were continuously tuned or calibrated to give a deformation gradient similar to the gradient obtained from preliminary finite element analysis (mesh shown in Fig. 9).

\section{RESULTS AND DISCUSSIONS}

The results from each block were then assembled and the final deformation gradient was obtained and shown in Fig. 10.

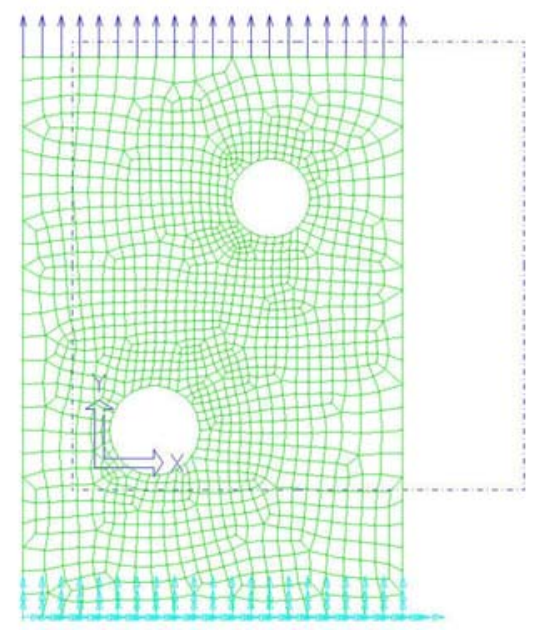

Figure 9: Finite element model of the test specimen.
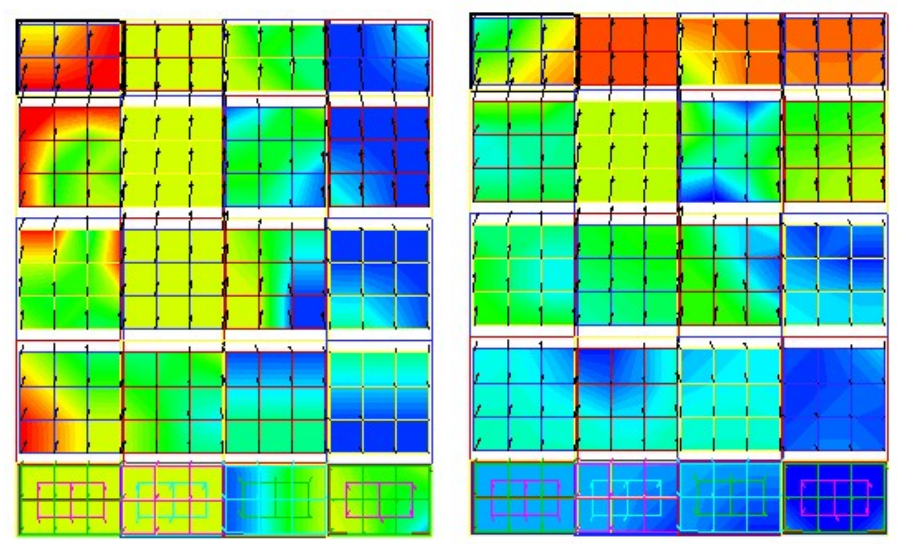

Figure 10: Displacement distribution of displacement components $u$ and $v$ from the genetic algorithm. 
Referring to Fig. 10, it is evident that the information at the interface between the different blocks is yet unknown and the elastostatic compatibility equations are not satisfied. This is achieved by computationally integrating the various blocks together by averaging the intensity of the nodes at the edges between neighboring blocks along the median coordinates between the edges, using the RBF interpolation technique. This resulted in a displacement distribution of the overall specimen as a single entity and not a compilation of several entities. Figs 11 and 12 exhibit the deformation distribution plots magnified by a factor of 10 as compared to the solution obtained from preliminary finite element analysis.

The strain components $\varepsilon_{x}, \varepsilon_{y}$ and $\gamma_{x y}$ were computed by the RBF-FD differentiation scheme and their distribution is shown in Fig. 13.
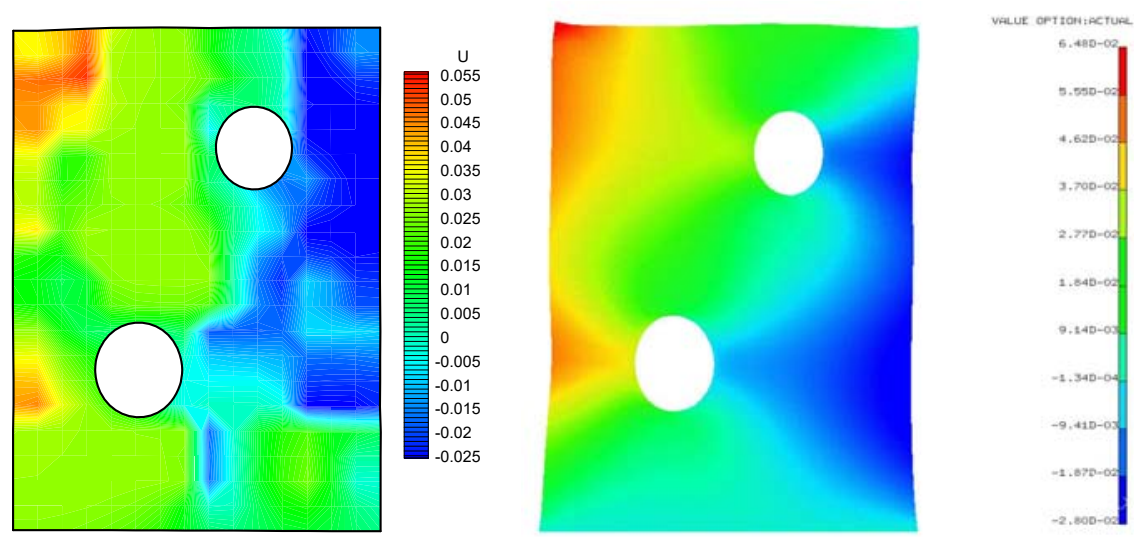

Figure 11: Displacement distribution of $u$ at 10X Magnification for the integrated genetic algorithm data as compared to solution obtained from FEA.
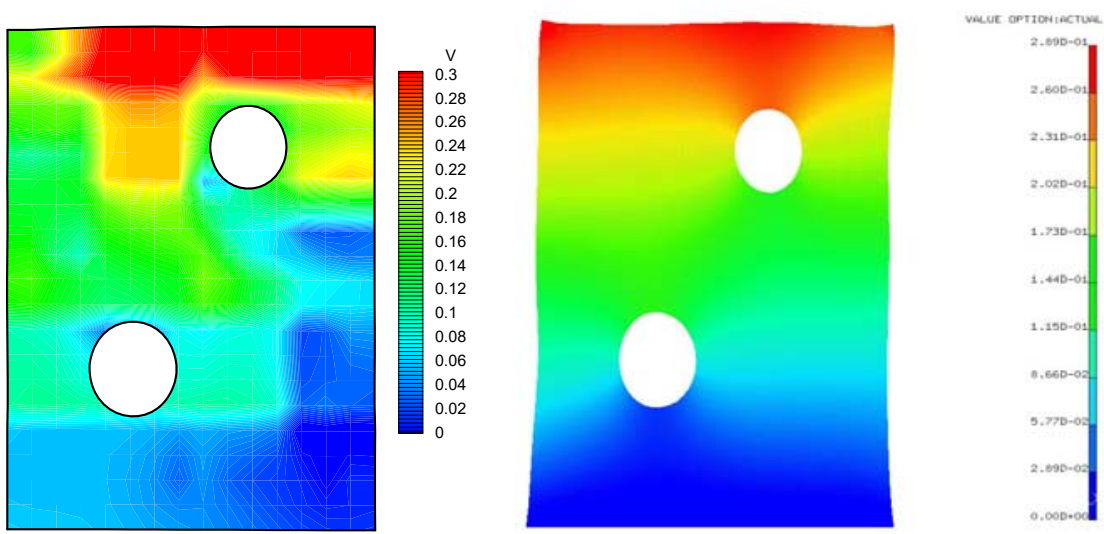

Figure 12: Displacement distribution of $v$ at 10X Magnification for the integrated genetic algorithm data as compared to solution obtained from FEA. 

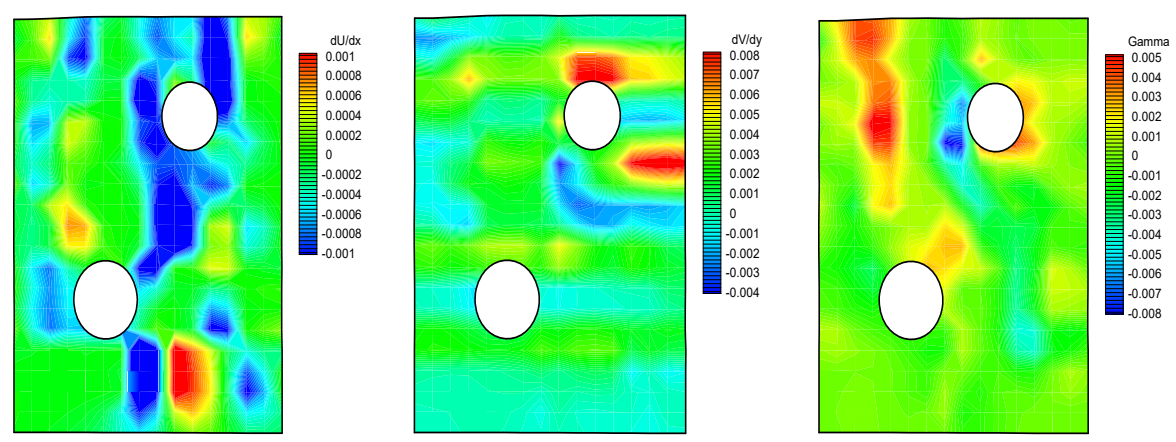

Figure 13: Strains components $\varepsilon_{x}, \varepsilon_{y}$ and $\gamma_{x y}$ distribution obtained by RBF interpolation.

\section{CONCLUSIONS}

The GA-based digital image correlation technique is successfully applied to measure surface displacements in structures using laser speckle photography. With readily available high-resolution digital cameras, the sensitivity of this technique can be significantly increased. A major advantage of this technique is its ability to subdivide the domain and hence use the full camera resolution on a small region. In addition, the GA was used to compute only the displacement components (two variables), which makes the technique more efficient. This is complemented by the RBF implementation to accurately compute the strain components. The technique is completely non-contact and highly accurate even for very small displacements.

\section{REFERENCES}

[1] Erf, R.K., Speckle Metrology, Academic Press: New York, 1978.

[2] Vest, C.M., Holographic Interferometry, John Wiley: New York, 1979.

[3] Kobayashi, A.S., Handbook on Experimental Mechanics, VCH Publishers: New York, 1993.

[4] Dainty, J.C., Laser Speckle and Related Phenomena, Springer-Verlag: New York, 1975.

[5] Fraley, J.E., Hamed, M.A., Peters, W.H. \& Ranson, W.F., Experimental boundary integral equation application in speckle interferometry. Proceedings 1981, SESA Spring Conference, pp. 68-71, 1981.

[6] Peters, W.H. \& Ranson, W.F., Digital imaging techniques in experimental stress analysis. Optical Engineering, 21(3), pp. 427-431, 1982. DOI: 10.1117/12.7972925.

[7] Pilch, A., Maudlin, J., Mahajan, A. \& Chu, T., Intelligent image correlation using genetic algorithms for measuring surface displacements and strain profiles. Proceedings of the 2001, ASME International Mechanical Engineering Congress and Exposition, DSC 70, pp. 81-88, 2001.

[8] Chu, T., Mahajan, \& Liu, C.T., An economical vision-based method to obtain wholefield deformation profiles. Experimental Techniques, 26(6), pp. 25-28.

DOI: 10.1111/j.1747-1567.2002.tb00087.x.

[9] Goldberg, D.E., Genetic Algorithms in Search, Optimization and Machine Learning, Addison-Wesley: Reading, MA, 1989. 
[10] Divo, E.A., Kassab, A.J. \& Rodríguez, F., An efficient singular superposition technique for cavity detection and shape optimization. Numerical Heat Transfer, Part B, 46(1), pp. 1-30, 2004.

[11] Hardy, R.L., Multiquadric equations of topography and other irregular surfaces. Journal of Geophysical Research, 176, pp. 1905-1915.

[12] Buhmann, M.D., Radial Basis Functions: Theory and Implementation, Cambridge University Press: Cambridge, 2003.

[13] Kansa, E.J. \& Hon, Y.C., Circumventing the Ill-conditioning problem with multiquadric radial basis functions: Applications to elliptic partial differential equations. Computers and Mathematics with Applications, 39(7-8), pp. 123-137, 2000. DOI: 10.1016/s0898-1221(00)00071-7.

[14] Pepper, D., Kassab, A. \& Divo, E., An Introduction to Finite Element, Boundary Element, and Meshless Methods: with Applications to Heat Transfer and Fluid Flow, ASME Press, 2014.

[15] Sarler, B., Tran-Cong, T. \& Chen, C.S., Meshfree direct and indirect local radial basis function collocation formulations for transport phenomena. Boundary Elements $X V I I$, eds A. Kassab, C.A. Brebbia \& E. Divo, WIT Press: Southampton and Boston, pp. 417-428, 2005. 